Publisher homepage: www.universepg.com, ISSN: 2663-7529 (Online) \& 2663-7510 (Print) https://doi.org/10.34104/ejmhs.020.061067

European Journal of Medical and Health Sciences

Journal homepage: www.universepg.com/journal/ejmhs

\title{
An In silico Approach for Structural and Functional Annotation of Uncharacterized Protein Rv0986 present in Mycobacterium tuberculosis
}

\author{
Abu Saim Mohammad Saikat ${ }^{1}$, Md. Lutful Kabir ${ }^{1}$, and Abul Bashar Ripon Khalipha ${ }^{2}$ \\ ${ }^{1}$ Dept. of Biochemistry and Molecular Biology, Bangabandhu Sheikh Mujibur Rahman Science and Technology University \\ (BSMRSTU), Gopalganj, Bangladesh; ${ }^{2}$ Dept. of Pharmacy, Bangabandhu Sheikh Mujibur Rahman Science and Technology \\ University (BSMRSTU), Gopalganj, Bangladesh. \\ *Correspondence: asmsaikat.bmb@gmail.com (Abu Saim Mohammad Saikat, Dept. of Biochemistry and Molecular Biology, \\ BSMRSTU, Gopalganj, Bangladesh.
}

\begin{abstract}
Tuberculosis (TB) is an ancient infectious disease caused by Mycobacterium tuberculosis (MTB). MTB is a human pathogen. Surprisingly, TB has become the top disease for its death rate worldwide. The uncharacterized protein Rv0986 is closely related to the transporters of the ATP-binding cassette domain, therefore, take part in the export of macrolide as well as a lipoprotein. Furthermore, it is associated with cell division protein. Hence, the protein has a significant role in mycobacterial infection. But, so far, the uncharacterized protein Rv0986 is not elaborated. As a result, in this study, the structural and functional annotation of the protein is described through in silico approach. The predicted tertiary structures of the protein generated by Swiss Model, Modeller, and Phyre2, and documented by the Ramachandran Plot analysis with PROCHECK, Verify 3D, and SwissModel Interactive Workplace. Z-score also applied for the overall structural assessment. This study will unleash the importance of the uncharacterized protein present in MTB, therefore, it provides an opportunity for drug and vaccine targeting against infection by MTB.
\end{abstract}

Keywords: Mycobacterium tuberculosis, Homology modeling, Protein Rv0986, In silico, and Ligand binding site.

\section{INTRODUCTION}

Mycobacterium tuberculosis is a human pathogen caused the most lethal disease tuberculosis. MTB is a rod-like, acid-fast, Gram-positive organism. Amazingly, TB is one of the most lethal diseases and ranked over HIV/AIDS globally. Tuberculosis spreads from infected people with MTB bacteria into the air, i.e. by coughing. In general, pulmonary $\mathrm{TB}$ causes by infection with lungs by MTB, and infection with MTB of other sites of the body causes extra pulmonary TB. It is estimated that about 10 million people are in new cases (range: 9.0-11.1 million), and about 1.2 million deaths by MTB infection (range, 1.1-1.3 million) even though people with HIV-negative in 2018. Therefore, people are at high risk for infection with MTB worldwide (WHO;Global tuberculosis report 2019; Ellison, 2019; Duarte et al., 2017). The protein Rv0986 is an ABC transporter ATP-binding protein of M. tuberculosis (strain ATCC 25618/H37Rv. Rv0986 has the ATPase catalytic subunit of an ABC transporter complex. This complex protein is deeply associated with coupling the energy of ATP hydrolysis which the main function is to the import of one or more from a variety of substrates like macrolide, lipoproteins, and hemin. As a result, Rv0986 is related to MTB infection (Wu et al., 2019; Holland and Holland, 2005; Beis, 2015). But, the protein structural and functional annotation with ligand binding active sites not explored. 
Therefore, the tertiary structure of the functional protein Rv0986 with detailed physicochemical characterization is predicted in this study by an in silico approach.

\section{MATERIALS AND METHODS}

\subsection{Sequence Retrieval}

The amino acid sequence of the functional protein Rv0986 collected from the UniProtKB server (Alex, 2019) following the accession ID of P9WQK1. There was no tertiary structure available of the protein Rv0986 in the Protein Data Bank. Hence, the tertiary structure modeling of the protein Rv0986 undertook by using the 248 amino acid long protein sequence.

\subsection{Physicochemical Characterization}

We used two web-based servers for the determination of the physicochemical properties of the uncharacterized protein Rv0986 present in MTB. ExPasy's ProtParam tool (ProtParam, 2017) used to predict the composition of amino acid, aliphatic and instability index, extinction coefficients, and GRAVY - a grand average of hydropathicity. Also, the isoelectric point (pI) predicted by the Sequence Manipulation Suite (SMS) tool of version 2 (Martin, 2016).

\subsection{Secondary Structure Prediction}

The self-optimized prediction method with alignment (SOPMA) (Combet et al., 2000) and SPIPRED (David, 1999) programs used for the secondary structure prediction of the protein Rv0986. DISOPRED tool (Vikram and Kumar, 2018) utilized for disorder prediction.

\subsection{Tertiary Structure Modeling and Validation}

Three web-based servers including Modeller (Zimmermann et al., 2018) following the HHpred (Zimmermann et al., 2018), Swiss Model (Arnold et al., 2020), and Phyre2 (Kelley et al., 2015) applied for tertiary structure prediction of the protein Rv0986 present in MTB.

In the homology protein modeling process, the most suitable template selected for final tertiary structures prediction. The Ramachandran map analysis following the PROCHECK and the Verify 3D server (https://servicesn.mbi.ucla.edu/Verify3D/) app-lied for the modeled tertiary structure quality documentation. Also, theSwiss-Model Interactive Work place (https://swissmodel.expasy.org/assess) followed for the structural quality assessment of the predicted protein structures. Also, the Prosa-web (Wiederstein and Sippl, 2007) used to obtain the Z-scores which was followed for the overall tertiary structural quality assessment experiment.

\section{RESULTS AND DISCUSSION}

\subsection{Physicochemical Characterization}

The protein Rv0986 is 248 amino acids long and the sequence retrieved in the FASTA format. This protein sequence applied as a query sequence to detect the physicochemical characteristics of the protein Rv0986 present in MTB. As the instability index is 32.44 which is less than 40, the protein is stable (Guruprasad et al., 1990; Islam et al., 2020). The theoretical isoelectric point ( $\mathrm{pI}$ ) indicates that the protein is acidic (pI 5.63, 5.72*).

The molecular weight of the protein Rv0986 is 27373.11 Da. The higher aliphatic index value of the protein is 95.52 indicating as a positive factor for increased thermos-stability for a wide temperature range (Gill, and Hippel, 1989). The hydrophilic nature of the protein and the possibility of better interaction with water (Ikai, 1980; Shahen et al., 2019) were indicated by the GRAVYindices value of -0.265 (Table 1).

\subsection{Secondary Structure Prediction}

SOPMA considered the default parameters including, window width: 17; similarity threshold: 8 ; division factor: 4, for secondary structure prediction of the protein Rv0986 present in MTB.

SOPMA utilized 248 amino acid-long protein sequences (sub-database) as well as 33 aligned proteins; therefore, it predicted $32.66 \%$ of the residues as random coils in comparison to alpha-helix of $47.18 \%$, Beta turn of $5.24 \%$, and the extended strand of $14.92 \%$ (Table 2). The PSIPRED program showed higher confidence in the prediction of the helix, strand, and coil (Fig 1). 
Table 1: Physicochemical Parameters.

\begin{tabular}{|l|l|}
\hline Physicochemical Parameters & Values \\
\hline Number of amino acids & 248 \\
\hline Molecular weight & 27373.11 \\
\hline Theoretical isoelectric point (pI) & $5.63,5.72^{*}$ \\
\hline Aliphatic index & 95.52 \\
\hline \begin{tabular}{l} 
The instability index (II) \\
\hline $\begin{array}{l}\text { Total number of negatively } \\
\text { charged residues (Asp + Glu) }\end{array}$
\end{tabular} & 32.44 \\
\hline $\begin{array}{l}\text { Total number of positively charged } \\
\text { residues (Arg + Lys) }\end{array}$ & 26 \\
\hline $\begin{array}{l}\text { Grand average of hydropathicity } \\
\text { (GRAVY) }\end{array}$ & -0.265 \\
\hline
\end{tabular}

Table 2: Secondary Structure Elements.

\begin{tabular}{|l|l|}
\hline $\begin{array}{l}\text { Secondary Structure } \\
\text { Elements }\end{array}$ & Values $(\%)$ \\
\hline Alpha helix $(\mathrm{Hh})$ & 47.18 \\
\hline $3_{10}$ helix $(\mathrm{Gg})$ & 0.00 \\
\hline Pi helix $(\mathrm{Ii})$ & 0.00 \\
\hline Beta bridge $(\mathrm{Bb})$ & 0.00 \\
\hline Extended strand $(\mathrm{Ee})$ & 14.92 \\
\hline Beta turn $(\mathrm{Tt})$ & 5.24 \\
\hline Bend region $(\mathrm{Ss})$ & 0.00 \\
\hline Random coil $(\mathrm{Cc})$ & 32.66 \\
\hline Ambiguous states & 0.00 \\
\hline Other states & 0.00 \\
\hline
\end{tabular}

*pI determined by SMS Version2

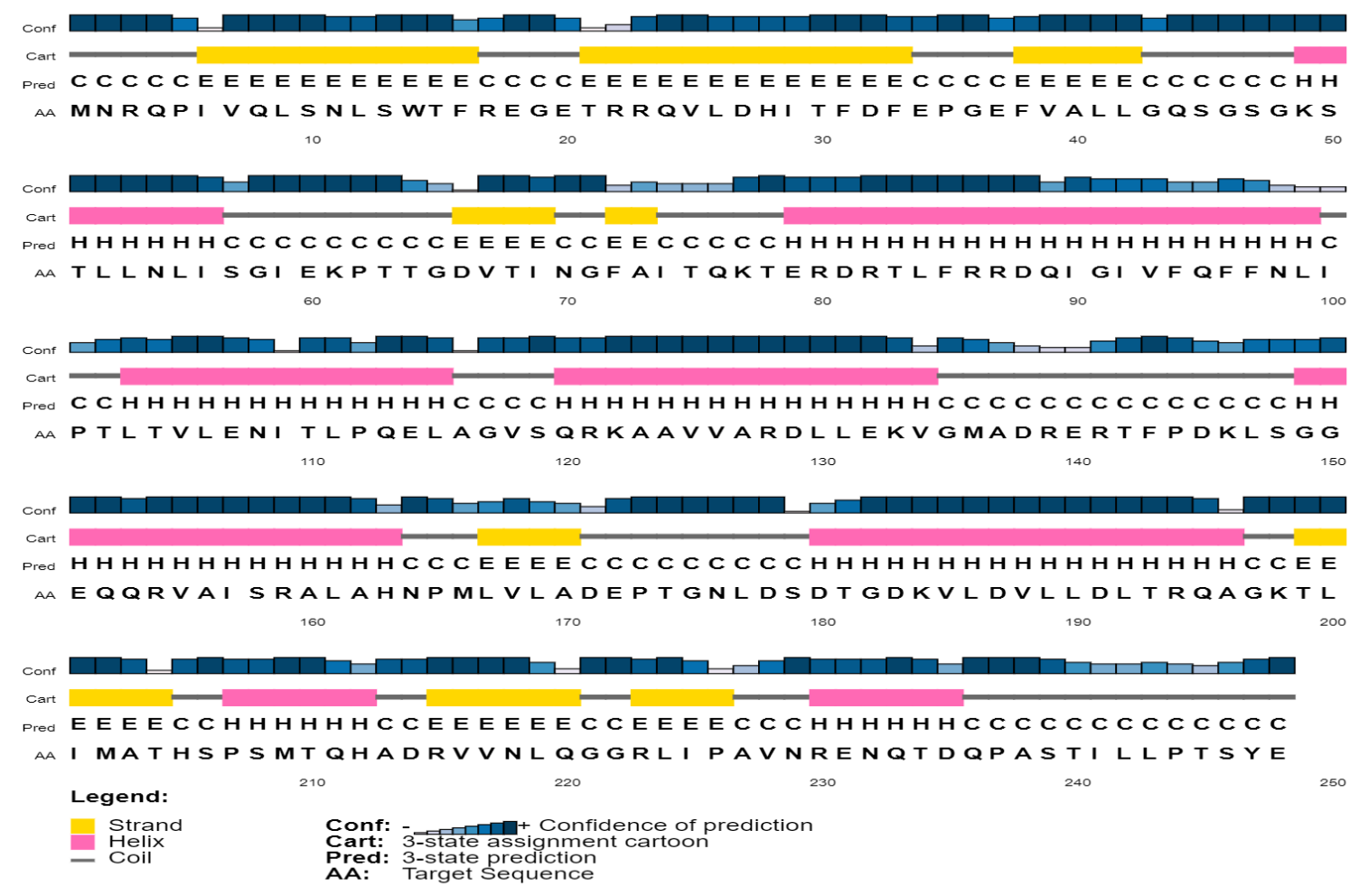

Fig 1: Predicted Secondary Structure.

\subsection{Protein-Protein and Protein-Polynucleotide Binding Sites}

Predict protein server predicted the binding sites in the protein structure of the protein Rv0986 where 11 different protein binding sites identified at the positions of: $1-4,17-22 ; 61-62 ; 84 ; 87-88 ; 120-122$; $132-133 ; 139-140 ; 193 ; 212 ; 229-234 ; 246$. There 4 different polynucleotide binding sites identified at the positions of: 45; 47; 49; and 49-50 (Fig 2).

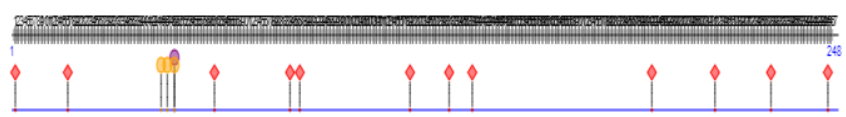

Fig 2: Protein-Protein and Protein-Polynucleotide Binding Sites. 


\subsection{Structure Modeling and Validation}

The HHpred tool was used for executing the Modeller for tertiary structure prediction. Protein sequence in FASTA format inserted into HHpred (Zimmermann et al., 2018) and executed the Modeller (Webb and Sali, 2016). The most suitable template selected (3TUI_D) among the number of hits of 250 for tertiary structure prediction of the protein Rv0986. This template indicated the probability rate of $100 \%$, SS of 27.5 , E-Value of 6.6e-32, Cols of 239, and the target length of 366 (data not shown). The generated structure stored in PDB format (Fig 3). The Ramachandran Map was analysis by PROCHECK (Table 3) indication an excellent protein model quality. It showing that $95.2 \%$ of the total residues found in the core; $4.8 \%$ of residues in the additional allowed regions. But, there was no residue in both generously allowed regions and the disallowed regions (Fig 5). The number of nonglycine and non-proline residues (209) was $100 \%$; 2 residues were as the end-residues (excl. Gly and Pro); the proline and glycine residues were 11 and 18, respectively, among the total residues (Fig 3). The Verify 3D tool documented the modeled tertiary protein structure (data not shown). The Swiss-Model Interactive Workplace used for the structural tertiary protein structure assessment experiment indication an excellent protein structure showing the MolProbity Score of 3.02; Ramachandran favored of $97.48 \%$ with the Qualitative Model Energy Analysis (QMEAN), $\mathrm{C} \beta$, All Atom, solvation, and torsion values of $1.15,-2.72,-3.06,0.18$, and -0.61 , respectively (data not shown).

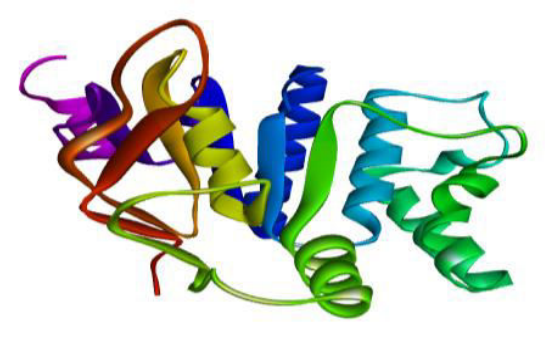

Fig 3: Structure of Rv0986 Predicted by Modeller
Similarly, the Phyre2 server was used for the prediction of the tertiary protein structure of the protein Rv0986. The tertiary structure predicted based on the top suitable template (c5ws4A) bearing the value of confidence of $100.0 \%$ and the coverage of $99 \%$ (Fig 4a). Phyre2 modeled 245 residues (99\% of the protein sequence) with $100.0 \%$ confidence. Phyre2 also described the secondary structure parameter including the disordered of $16 \%$, beta-strand of $25 \%$, alpha-helix of 39\% (data not shown). The Ramachandran Map followed by the PRO-CHECK indicating $87.9 \%$ of the residues in most favored regions and $11.2 \%$ as in additional allowed regions. There $0.5 \%$ of the total residues were in the disallowed regions, and $0.5 \%$ residues in the generously allowed regions (Table 3). This modeled the tertiary structure of the protein Rv0986 passed the Verify 3D protein structural quality assessment experiment (data not shown). The Swiss-Model Interactive Workplace validated the modeled protein structure by indicating the values of the MolProbity Score of 2.8 , as well as the Ramachandran, favored of 91.80\%; the QMEAN, C $\beta$, all-atom, solvation, and torsion values of $-1.92,-1.81,-1.88,0.54$, and 1.81 , respectively (data not shown).

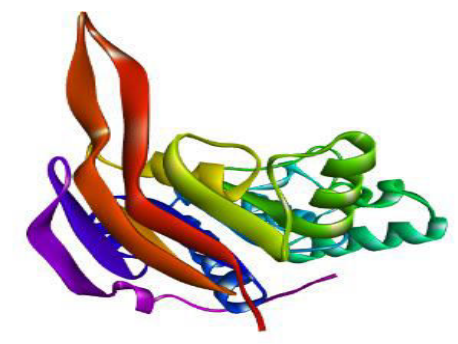

Fig 4 (a): Structure of Rv0986 Predicted by Phyre2.

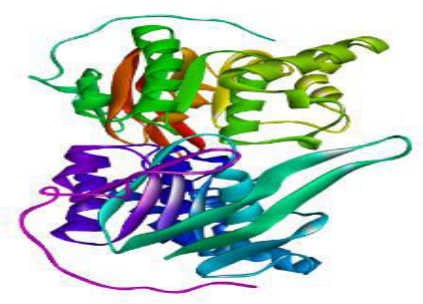

Fig 4 (b): Structure of Rv0986 Predicted by Swiss Model. 
Table 3: Ramachandran Plot Analysis.

\begin{tabular}{|l|l|l|}
\hline Server & Ramachandran Plot Calculation & Value (\%) \\
\hline \multirow{4}{*}{ Modeller } & Residues in most favored regions [A,B,L] & 95.2 \\
\cline { 2 - 3 } & Residues in additional allowed regions [a,b,l,p] & 4.8 \\
\cline { 2 - 3 } & Residues in generously allowed regions [ a, $\sim \mathrm{b}, \sim 1, \sim \mathrm{p}]$ & 0.0 \\
\cline { 2 - 3 } Phyre2 & Residues in disallowed regions & 0.0 \\
\hline \multirow{5}{*}{ Swiss Model } & Residues in most favored regions [A,B,L] & 87.9 \\
\cline { 2 - 3 } & Residues in additional allowed regions [a,b,l,p] & 11.2 \\
\cline { 2 - 3 } & Residues in generously allowed regions [ a, $\sim \mathrm{b}, \sim 1, \sim \mathrm{p}]$ & 0.5 \\
\cline { 2 - 3 } & Residues in disallowed regions & 0.5 \\
\cline { 2 - 3 } & Residues in most favored regions [A,B,L] & 92.9 \\
\cline { 2 - 3 } & Residues in generously allowed regions [ a, $\sim \mathrm{b}, \sim 1, \sim \mathrm{p}]$ & 6.4 \\
\cline { 2 - 3 } & Residues in disallowed regions & 0.5 \\
\hline
\end{tabular}

Likewise, the tertiary structure of the protein Rv0986 also modeled by the Swiss-Model server. The 3D structure modeled based on the most suitable template selected by comparing with the top five similar level templates (5lj6.1.A, 5lil.1.B, 5lil.1.A, 5lil.1.B, and 2ouk.5.A). The selected template parameters were including the Qualitative Model Energy Analysis (QMEAN) score of (-1.03), Global Model Quality Estimate (GMQE) score of 0.73 , percentage of sequence identity of 39.50, and the coverage of $96 \%$. Generated protein structure stored in PDB file format. The Ramachandran Map by PROCHECK showed that $92.9 \%$ of the total residues found in the, and $92.9 \%$ of residues in the additional allowed regions. There were $0.5 \%$ of residues in the generously allowed regions and $0.2 \%$ of the residues in the disallowed regions (Table 3). In the predicted protein structure, the number of nonglycine and non-proline residues was 423 which was $100 \%$ and 4 residues were asthe end-residues (excl. Gly and Pro). Among the total residues in the protein structure, the glycine residues and proline residues were 36 and 22, respectively (Fig 4b). Verify 3D validated the protein structural quality as an excellent structure of the protein (data not shown). The Swiss-Model Interactive Workplace validated the modeled tertiary structure of the protein Rv0986 indicating as the
MolProbity Score of 1.53 and the Ramachandran favored $96.88 \%$. The values for the predicted tertiary structure are including the QMEAN (Qualitative Model Energy Analysis), C $\beta$, All Atom, solvation, and torsion values of -1.03, $2.87,-1.07,0.10$, and -0.53 , respectively (data not shown).

The Prosa-web (Wiederstein and Sippl, 2007) server used for the overall tertiary structure quality assessment. The Prosa-web calculated the Standard Bond Angles and Z-scores. The Z-scores used to estimate the 'degree of nativeness' of the predicted structures (Nas et al., 2020). The Zscores for the modeled tertiary structures by the three servers including Modeller, Phyre2, and Swiss-Model were -7.42, -7.31, and -7.37, respectively. In this paper, Modeller, Phyre2, and Swiss-Model servers are presenting similar values.

\section{CONCLUSIONS}

In this study, it is concluded that the protein Rv0986 present in Mycobacterium tuberculosis is a functional protein, thereby, associated with microbial infection in humans. As the protein tertiary structure not available in the Protein Data Bank, the structural model prediction with its structural and functional annotation is well explained in this paper. Hence, provides an insight 
into this uncharacterized protein Rv0986. This study will bolster and sharpen our knowledge in pathogenesis, therefore, unleashes an opportunity for drug and vaccine targeting against the infection by MTB.

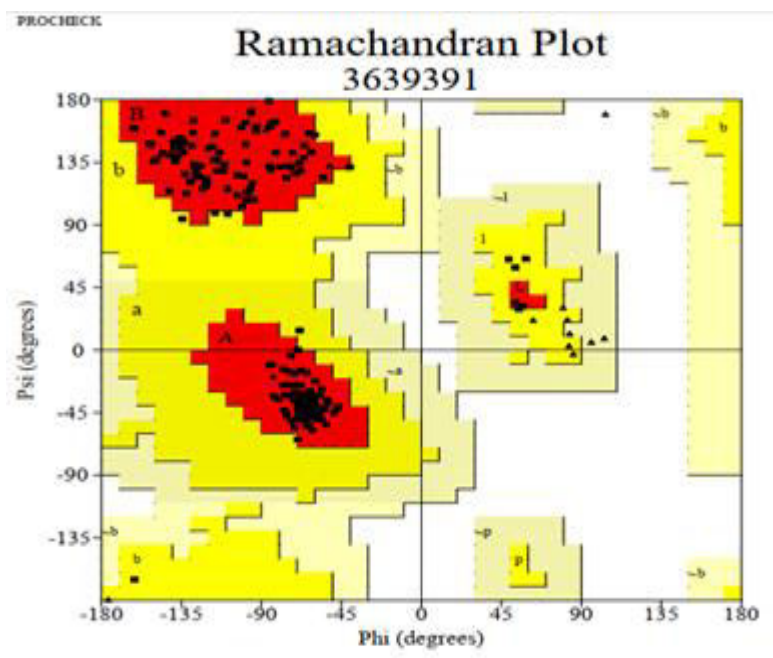

Fig 5: Ramachandran Plot Analysis.

\section{ACKNOWLEDGEMENTS}

We would like to thank the Dept of Biochemistry and Molecular Biology, and the Dept. of Pharmacy of Bangabandhu Sheikh Mujibur Rahman Science and Technology University, Gopalganj, Bangladesh, for supporting this study.

\section{CONFLICTS OF INTETEST}

The author declares no conflict of interest.

\section{REFERENCES}

1. Alex Bateman (2019). 'UniProt: a worldwide hub of protein knowledge', Nucleic Acids Research, 47(D1), D506-D515 https://doi.org/10.1093/nar/gky1049

2. Arnold, K. et al. (2020). 'The SWISSMODEL workspace: a web-based environment for protein structure homology modelling', Bioinformatics, 22(2), 195-201 https://doi.org/10.1093/bioinformatics/bti770

3. Beis K. (2015). 'Structural basis for the mechanism of ABC transporters', Biochemical Society Transactions. Portland Press Ltd, 43, 889-893.

https://doi.org/10.1042/BST20150047
4. Combet C, Blanchet C, Geourjon C, Deléage G. (2000). 'NPS@: network protein sequence analysis' (2020), COMPUTER CORNER, 25(3), 147-150.

https://doi.org/10.1016/S0968-0004(99)01540-6

5. David T. Jones, (1999). 'Protein secondary structure prediction based on position-specific scoring matrices', Journal of Molecular Biology, 292(2), 195-202

https://doi.org/10.1006/jmbi.1999.3091

6. Duarte T. A. et al. (2017). 'A systematic review of East African-Indian family of Mycobacterium tuberculosis in Brazil', Brazilian J. of Infectious Diseases, 21(3), 317-324.

https://doi.org/10.1016/j.bjid.2017.01.005

7. Ellison A. L. (2018). 'Exploring the role of the striengent respose in by'.

8. Gill C, Hippel P. H. von. (1989). 'Calculation of protein extinction coefficients from amino acid sequence data', Analytical Biochemistry, 182(2), 319-326.

https://doi.org/10.1016/0003-2697(89)90602-7

9. Guruprasad K. et al. (1990). Correlation between stability of a protein and its dipeptide composition: a novel approach for predicting in vivo stability of a protein from its primary sequence, Protein Engineering, Design and Selection, 4(2), 155-161. https://doi.org/10.1093/protein/4.2.155

10. Holland K. A. and Holland, I. B. (2005) 'Adventures with ABC-proteins: Highly conserved ATP-dependent transporters', Acta Microbiologica et Immunologica Hungarica, 52(3-4), 309-322.

https://doi.org/10.1556/amicr.52.2005.3-4.4

11. Ikai A. (1980) Thermostability and Aliphatic Index of Globular Proteins, The Journal of Biochemistry, 88(6), 1895-1898.

https://doi.org/10.1093/oxfordjournals.jbchem. $\underline{\mathrm{a} 133168}$

12. Islam S, Saleh MA, ShahriarZaman. (2020). Molecular identification and characterization of smartphone screen associated pathogenic bacteria. Am. J. Pure Appl. Sci., 2(3), 94-103. https://doi.org/10.34104/ajpab.020.0940103

13. Kelley L. A. et al. (2015) 'The Phyre2 web portal for protein modeling, prediction and analysis', Nature Protocols. Nature Publishing Group, 10(6), 845-858.

https://doi.org/10.1038/nprot.2015.053 
14. Martin L. (2016) 'Genomics and transcriptomics of the molting gland (Y-organ) in the blackback land crab, Gecarcinus lateralis'. Colorado State University, M.Sc Thesis, 1-97. http://hdl.handle.net/10217/176669

15. Nas FS, Yahaya A, and Ali M. (2020). Prevalence of Trichomonas vaginalis among pregnant women attending ante-natal care in Kano, Nigeria. Eur. J. Med. Health Sci., 2(2), 39-45.

https://doi.org/10.34104/ejmhs.020.39045

16. ProtParam, E. (2017). 'ExPASy-ProtParam tool'.

17. Shahen MZ, Mahmud S, Uddin ME and Alam MS. (2019). Effect of antibiotic susceptibility and inhibitory activity for the control of growth and survival of microorganisms of extracts of Calendula officinalis, Eur. J. Med. Health Sci. 1(1), 1-9. https://doi.org/10.34104/ejmhs.019

18. Vikram, T., Kumar P. (2018). 'Analysis of Hepatitis E virus (HEV) X-domain structural model', Bioinformation. 14(7): 398-403. https://doi.org/10.6026/97320630014398

19. Webb B. and Sali, A. (2016) 'Comparative
Protein Structure Modeling Using MODELLER', Current Protocols in Protein Science. 86(1), 2.9.1-2.9.37.

https://doi.org/10.1002/cpps.20

20. Wiederstein M, and Sippl M. J, (2007), ProSA-web: interactive web service for the recognition of errors in three-dimensional structures of proteins, Nucleic Acids Research, 35(suppl_2), W407-W410. https://doi.org/10.1093/nar/gkm290

21. WHO; Global tuberculosis report 2019 (2019). Tuberculosis (TB), 1-297. https://www.who.int/tb/publications/global_re port/en/

22. Wu C. et al. (2019) 'Insect ATP-binding cassette $(\mathrm{ABC})$ transporters: Roles in xenobiotic detoxification and $\mathrm{Bt}$ insecticidal activity', Int. J. Mol. Sci., 20(11), 2829. https://doi.org/10.3390/ijms20112829

23. Zimmermann, L. et al. (2018) 'A Completely Reimplemented MPI Bioinformatics Toolkit with a New HHpred Server at its Core', J. of Molecular Biology. Academic Press, 430(15), 2237-2243.

https://doi.org/10.1016/j.jmb.2017.12.007

Citation: Saikat ASM, Kabir ML, and Khalipha ABR. (2020). An In silico approach for structural and functional annotation of uncharacterized protein Rv0986 present in Mycobacterium tuberculosis, Eur. J. Med. Health Sci., 2(3), 61-67. https://doi.org/10.34104/ejmhs.020.061067 C) () 